\title{
Contrôle génétique des différents types de fibres musculaires
}

\author{
Matthieu Dos Santos, Iori Sakakibara, Frédéric Auradé, Maud Wurmser, Stéphanie Backer, \\ Marcio Do Cruzeiro, Jean Paul Concordet, Daan Noordermer, Frédéric Relaix, Pascal Maire
}

Les muscles squelettiques sont composés de fibres à vitesse de contraction lente ou rapide. Il existe trois sous-types de fibres rapides, chacun exprimant une isoforme différente de chaîne lourde de la myosine (MYH) codée par trois gènes distincts situés au même locus [1]. Le contrôle spatio-temporel de l'expression de ces gènes n'est pas connu. Notre objectif est d'étudier le lien entre l'organisation 3D de la chromatine du locus Myh et l'expression de ces gènes dans les fibres musculaires.

Nous avons créé un modèle de souris transgénique à partir d'un BAC (Bacterial Artificial Chromosome) d'environ $250 \mathrm{~kb}$ du locus Myh rapide ou nous avons cloné différents gènes rapporteurs fluorescents au niveau des premiers exons de chaque gène Myh rapide. Ce BAC a été injecté dans des ovocytes de souris et deux lignées de souris ont été obtenues: une lignée ayant intégré 2 copies complètes du BAC $\left(\mathrm{Enh}^{+}\right)$et une autre avec une délétion de la région 5' (Enh). Dans la lignée Enh', l'expression des transgènes récapitule celle des gènes endogènes. L'expression des transgènes dans la lignée Enh' est fortement réduite : la région 5' du BAC absente dans ces souris, est nécessaire pour contrôler spatialement l'expression des trois gènes rapides Myh.

Pour identifier ces éléments régulateurs, nous avons réalisé des expériences de Chromatin Conformation Capture (4C-seq) à partir de muscles squelettiques adultes. Ces expériences permettent d'identifier les interactions physiques entre un promoteur et des séquences régulatrices. Nous avons identifié une séquence régulatrice en cis d'environ $40 \mathrm{~kb}$ qui interagit avec le promoteur Myh4 au niveau du quadriceps et le promoteur Myh2 au niveau du soleus. Cette séquence pourrait être l'association de multiples séquences régulatrices; un SuperEnhancer. Son absence dans la lignée BAC Enh suggère que cette séquence est nécessaire pour activer et restreindre l'expression d'un seul gène de Myh rapide. Nous sommes en train de mieux caractériser les propriétés de ce SuperEnhancer dans la régulation des gènes de Myh rapides en générant des souris possédant une délétion de cet élément grâce à la technique de Crispr/Cas9 (Figure 1).

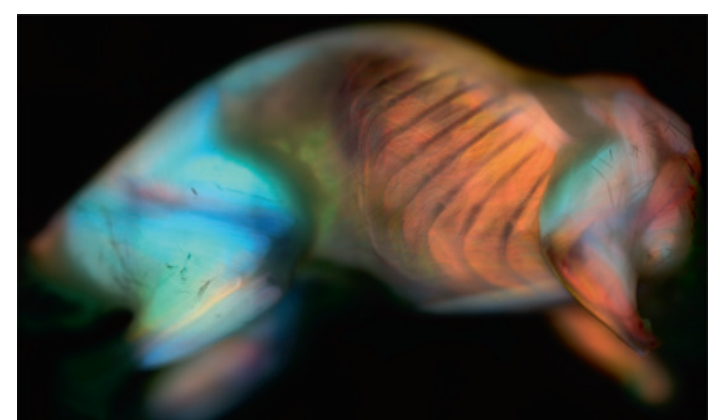

Figure 1

Image d'une souris transgénique 5 jours après la naissance, exprimant différents transgènes au niveau des différents types de fibres rapides. Les fibres bleues expriment Myh4, les fibres rouges Myh1 et les fibres vertes Myh2 (๑ photo : Matthieu Dos Santos, Institut Cochin).

Les fibres musculaires squelettiques sont des cellules plurinucléées pouvant posséder plus de 250 noyaux chez la souris. Moins de $5 \%$ des fibres adultes expriment plusieurs isoformes de Myh au niveau protéique [2,3], ce sont des fibres hybrides. Ces résultats suggèrent que dans $95 \%$ des fibres adultes, un seul gène Myh rapide du locus est activé et qu'un noyau ne peut exprimer qu'un seul gène de Myh rapide à la fois. Pour tester cette hypothèse, nous avons isolé des noyaux de muscle lent et rapide et nous avons séquencé les ARNm de chaque noyau individuellement grâce à la technique de Single Cell RNAseq de 10X genomics. Les résultats montrent que moins de $5 \%$ des noyaux expriment 2 isoformes à la fois. Pour confirmer cela et visualiser si les noyaux d'une myofibre ont une expression de Myh coordonnée, nous avons visualisé l'expression des premARN de Myh rapide par hybridation in situ à fluorescence (RNascope) sur des fibres musculaires isolées. Dans la majorité des cas un seul gène Myh rapide est exprimé par noyau et les différents noyaux d'une myofibres expriment la même isoforme de Myh. Cependant, dans l'EDL, environ $5 \%$ des fibres possèdent une décoordination d'expression: dans une même myofibre, certains noyaux expriment différentes isoformes et d'autres expriment deux gènes de Myh en même temps. Le 
nombre de fibres hybrides augmente de 10 fois au cours de la dénervation, prouvant que l'innervation participe à la coordination de l'expression des gènes de Myh rapide dans les myofibres.

Genetic control of skeletal muscle fiber type

\section{LIENS D'INTÉR̂̂T}

Les auteurs déclarent n'avoir aucun lien d'intérêt concernant les données publiées dans cet article.

\section{RÉFÉRENCES}

1. Schiaffino S, Reggiani C. Fiber types in mammalian skeletal muscles. Physiol Rev 2011 ; 91 : 1447-531.

2. Sawano S, Komiya Y, Ichitsubo R, et al. A one-step Immunostaining method to visualize rodent muscle fiber type within a single specimen. PLoS One 2016 ; 11 : e0166080.

3. Rowan SL, Rygiel K, Purves-Smith FM, et al. Denervation causes fiber atrophy and myosin heavy chain co-expression in senescent skeletal muscle. PLoS One 2012 ; E7 : e29082.

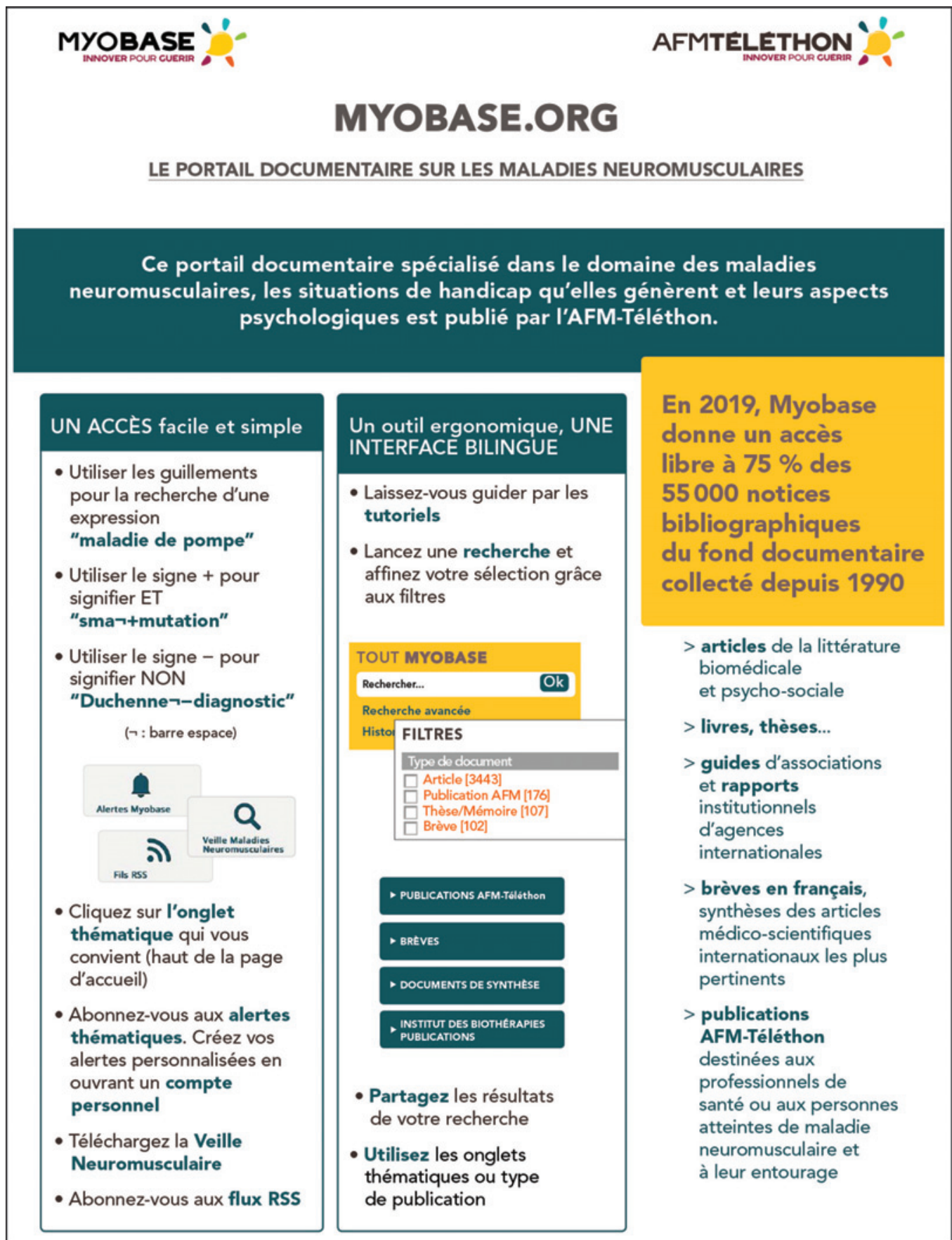

